\title{
SERVICE AND NETWORK MODELING FOR IMPLEMENTATION OF POWERLINE REMOTE MONITORING OF ELECTRICAL POWER METERING IN TANZANIA
}

\author{
J. Anatory and N.H. Mvungi \\ Department of Computer and Systems Engineering \\ Faculty of Electrical and Computer Systems Engineering \\ University of Dar es salaam, \\ P.O.Box 35131 Dar es Salaam, Tanzania \\ M. M. Kissaka \\ Department of Telecommunications Engineering \\ Faculty of Electrical and Computer Systems Engineering \\ University of Dar es salaam, \\ P.O.Box 35131 Dar es Salaam, Tanzania
}

\begin{abstract}
7 he conventional metering, preparment and estimates have been used in power utilities industries. The systems have experienced many problems such as power tampering, bill tampering, failure to receive invoices by power utility customers and so on. Different technologies have been investigated to overcome the problems and recently it has been realized that remote monitoring systems through powerline channel is a solution of these problems in Tanzania. However, the powerline network has been used for electrical power to use it for implementation of remote monitoring; network must be setup and has to be modeled to accommodate communications.
\end{abstract}

The contribution has analyzed the communication through powerline network from customer premises with two regenerative repeaters and CSMA/CD MAC protocol to distribution transformer. The modulation used is direct sequence spread spectrum with binary phase shift keying. The coding is Bose Chaudhuri Hacknguem (BCH). From distribution transformer to primary router at primary substation token bus is used. The wireless networks have been connected to PLC at primary router. Simulation results are presented.

Keywords: Metering technologies, powerline communications, medium access control, routers, traffic modeling

\section{INTRODUCTION}

Power utility companies have been using three systems to monitor and record energy consumed by customers. The systems are conventional metering, estimates and prepaid metering. Like other utility companies elsewhere-utilizing similar systems, developing countries companies experiences some problems. The problems are firstly customer failure to receive monthly bills at right times, which leads to in appropriate power disconnections for non payment of bills. Reactions to these problems include power theft. It has been estimated that loss due to this type of metering in Tanzania is USD 6,250 per day (Seger, 2002). In addition revenue losses (theft or fraud) are estimated to be approximately $35 \%$ of sales or USD 200 millions per annum. Many utility companies at the beginning was expected that prepayment meter will overcome the problem but today it has estimated that in Tanzania the loss duc to this type of meter is $30 \%$ of revenue collected through such kind of 
meters, also the scenario is the same in other countries such as South Africa, Mozambique, Ghana and others.

In developed countries the loss in power utility companies such as USA was USD 40million a year, Australia USD 15million but to-date companies have implemented remote monitoring systems which is based on sending and receiving metering data through telephone lines, radio telecommunication network (RTN), advanced wireless Network such as GSM and Powerline network. In the countries such as USA, Russia, China, Venezuela and others, different transmission media to be used in implementation of remote monitoring have been researched, it has been observed that for power utility companies the powerline channel is the leading transmission media in use due to availability of infrastructure at each customer premises, security issues and management. In addition the future promises of PLC as data transmission channels. PLC channel is in use for metering data transmission in developed countries, but all systems are very expensive and developed for those countries. It has been identified that IP based network is a promise cost effective for implementing Remote Monitoring through powerline network in Tanzania (Anatory, 2003). The typical powerline network of Tanzania is as shown in Figure 1. The parameter CM is the customer meter, DT is the distribution transformer.

The access segment of Power line network topology is a bus topology. All the customer meters are connected in parallel to a common power line media. This results in every transmission by any customer meters to be received by every other meter. To have successful transmission in powerline channel, the channel allocation among the competing stations is critical for proper operation of the network. The schemes allocated for proper operations of shared channel are called multiple accesses control. This can also be referred as media access control (MAC) protocol. Various researchers have researched the MAC protocols for power line channel.

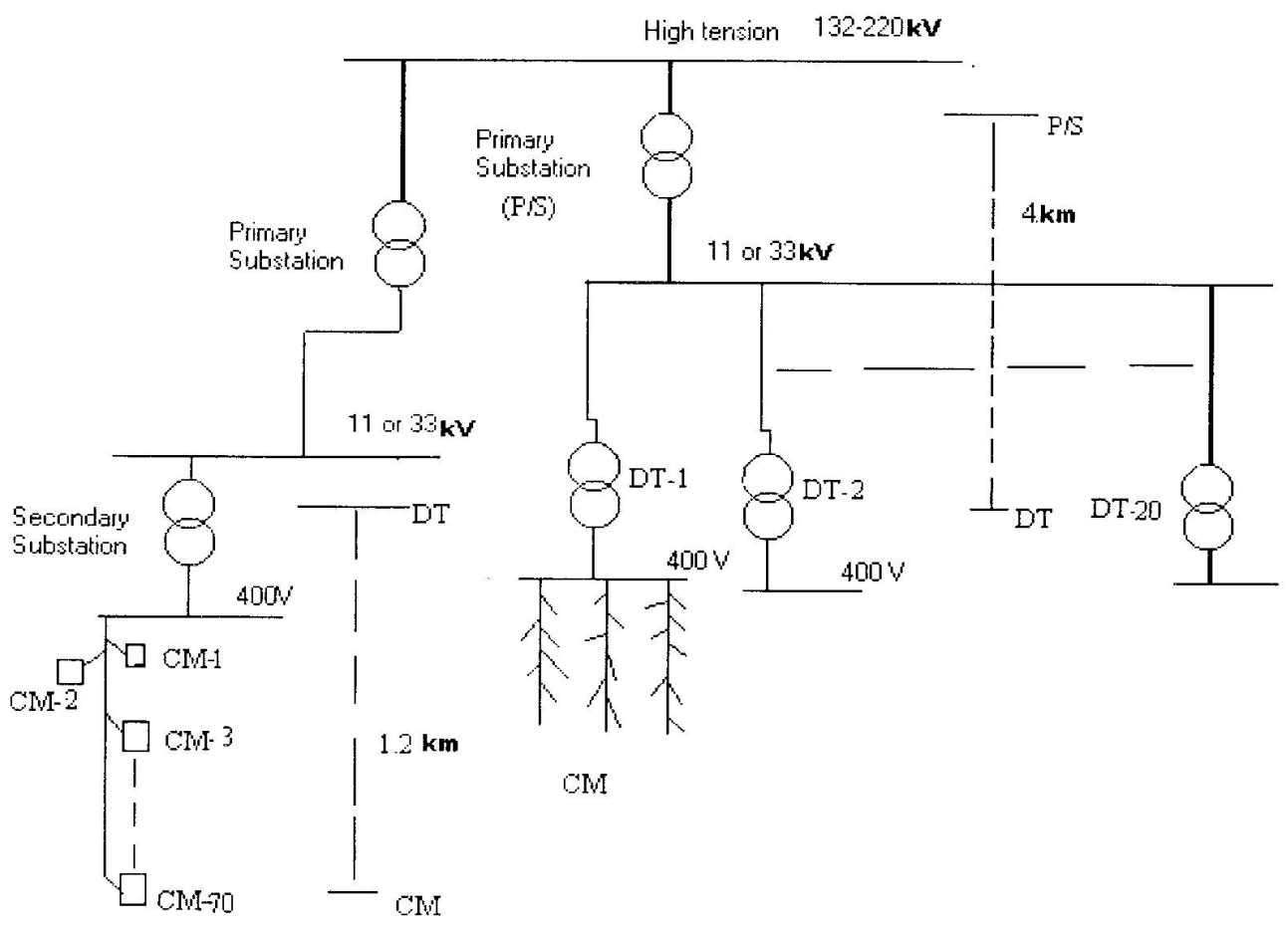

Figure 1: Powerline Network layout 
The MAC, which is considered to be used for power line channel, is classified into two parts; these are contention-based protocols and contentionless based protocols. The contentionbased protocols considered are ALOHA type and Carrier Sense Multiple Access with Collision Detections CSMA/CD (Onunga et al, 1989). The contentionless (Collision free) protocols, which have been considered for power line channel are polling and token bus. Recently, researchers have pointed out other feasible MAC for PLC. These include orthogonal frequency division multiple access (OFDMA), code division multiple access (CDMA), Time division multiple access (TDMA) and Frequency division multiple access (FDMA) (Anatory et al, 2003).

There are several criteria used to select MAC for given services. These are Traffic, service definitions, expected throughput and cost of implementation. The contention-based protocols are easy to implement but their throughput is very low compared to contentionless based protocol. Hence, this paper will select the required MAC based on traffic definition; throughput and easiness to implement in power line environment will be selected. It has been observed that, contention based MAC protocols are easily to implement. Therefore, contention based MAC can be used from end users to distribution transformer, while from distribution transformer, contentionless token bus protocol is more appropriate. This choice is based on the easy implementation of token bus compared to other contentionless protocols. The investigation which have already been done in the network of Tanzania, has pointed out that from customer meter (cm) to DT two hops are required using DSSS-BPSK and $\mathrm{BCH}$ code. This is with processing gain 14, SNR per bit $70 \mathrm{~dB}$ from $\mathrm{CM}$ to $\mathrm{DT}$. The traffic Figures have been estimated based on the problems faced power utility in developing countries like Tanzania.
Section two is explaining the proposed network architecture; section three is the traffic estimates. Section four is the metering traffic analysis and section five is the simulation results, then conclusion in section six.

\section{PROPOSED NETWORK ARCHITECTURE}

The design of the interface parts of a PLC energy meter and computer/router to powerline network in consideration is the direct sequence spread spectrum with Binary Phase Shift Keying (BPSK) as carrier modulation and $\mathrm{BCH}$ code. Carrier Sense Multiple Access with Collision Detections (CSMA/CD) and ALOHA systems is considered for access part from customer meters to distribution transformers. From the distribution transformers, token bus has been used by the bridging routers to route the packets towards the destinations upon receiving the token. At this part, the cyclic services like polling model have been considered. From customer meters to the bridging routers, this part is sub-netted. A single bridging router services one subnet. Two hops have been considered from customer meters to bridging routers to overcome attenuation in powerline channel.

Figure 2 gives a conceptual diagram of customer meters $(\mathrm{CM})$ communicating with the primary substation side of a distribution transformer through the bridging router at the distribution transformer.

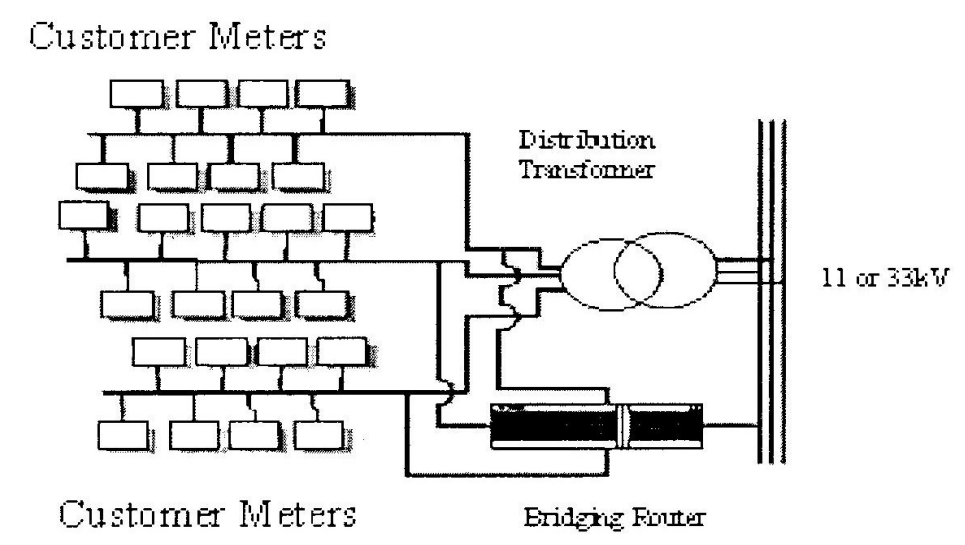

Figure 2: Power-Line Energy Monitoring Network Layout from Customer Meter to Bridging Router at Distribution Transformer (DT) 
Figure 3 is a network for different sites, with N1, $\mathrm{N} 2$ and N3 subnets. The routers are attached at single line on HT to overcome network loading. $\mathrm{SR}$ is a service router for network reliability. In case of failure in any phase of high voltage grid, data from bridging routers can be routed through SR, which will carry a traffic load of two phases. The primary router is installed on the primary substation side to control the entire network and sends the received packets to administrator server/Computer (AS/C). In this chapter metering traffic in powerline network from customer meters to where the $\mathrm{AS} / \mathrm{C}$ is located through IP based powerline networks is investigated.

11 or $33 k \mathrm{k}$ Liries

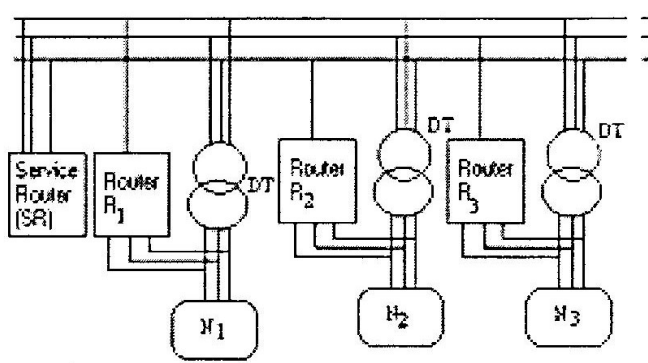

Key.

$\begin{array}{ll}\mathrm{N} 1, \mathrm{~N} 2, \mathrm{~N} & : \text { Swnets } \\ \mathrm{R}, \mathrm{R} 2 \mathrm{R} & \text { : Bndging Ronters } \\ \mathrm{SR} & \text { : Service Fouter } \\ \mathrm{AS} / \mathrm{C} & : \text { Adninistrator }\end{array}$

Figure 3: Layout of energy monitoring data network from different sites

\section{TRAFFIC ESTIMATES}

Data traffic estimates in a communication network can be obtained satisfactorily through measurements, which can be done when the network in consideration is in place. If the network does not exist, two methods can be adopted. These are detailed itemized services study or use of similar network for projection. In this research the service itemized method has been adopted. The number of utility customers connected at each phase is estimated. Hence, the projection of metering traffic intensity per customer meter is based on services included in customer meter design necessary to combat metering problems faced by power utility companies as explained in chapter one and two. The traffic estimates are used to predict the traffic handling capacity of each phase in a powerline network. Provision of the services considered a Bit Error Rate (BER) of $10^{-6}$ per packet length.

\section{Itemized Metering Services}

The energy meter at the power utility customer premises is expected to perform the following functions under worst-case conditions.

- Billing customer remotely once per month (BC)

- Disconnection of power at customer premises once per month (DM)

- Meter re-connection once per month (MC)

- Meter tampering detection and send the results 1-10 times per month (MT)

- Send and receive information on prepaid meters once per month (SRP)

- Meter reading once per week (MR)

- Power quality measurement and send the results 1-10 times per week (PQ)

- Alarm -information twice per month. (AI)

These have been used in traffic calculation and network capacity and determination of other parameters.

\section{Metering Data Traffic Estimation}

The assumption used in calculating metering data traffic is based on (Grabowski et al, 1989) that one A4 page is equivalent to $400 \mathrm{kbits}$. For metering communication purposes the information content is considered to be half of A4 page i.e. $200 \mathrm{kbits}$. The assumption made is that, the activities performed once per month and per week are performed simultaneously at the end of each month within working hours 
(w.hours) of a day and this will form busiest hours data traffic within a month. The data traffic (Ratel) generated within that period per second per meter is given by Equation (1). In addition, other activity such as tampering and power quality, which occurs in various days per month and week bases, contributes some data traffic to the busiest hours of a month. Those contributions of each term have been distributed within the month and weekly respectively to get the actual contribution to the network busiest hours. The data traffic rate (Rate2) and (Rate3) contributed by meter tampering (MT) and power quality (PQ) terms is as in equation (2) and equation (3) respectively. The total data traffic rate per meter $\left(D_{R T}\right)$ have been obtained by the contribution obtained from equation (1), (2) and (3).

$$
\begin{aligned}
\text { Rate }_{1}= & \frac{(B C+D M+M C+S R P+M R+A I) \text { bits } / \text { day }}{(\text { whour } / \text { day })(\mathrm{min} / \mathrm{hr})(\mathrm{sec} / \mathrm{min})} \\
& =\frac{(1+1+1+2+1+1)(200 \mathrm{kbits}) / \mathrm{day}}{(8 \mathrm{hrs} / \text { day })(60 \mathrm{~min} / \mathrm{hr})(60 \mathrm{Sec} / \mathrm{min})} \\
& =48.61111 \text { bits } / \mathrm{Sec} \\
\text { Rate }_{2}= & \frac{\text { MT bits } / \text { month }}{(\text { days } / \text { month })(\text { hours } / \text { dav })(\mathrm{min} / \text { hour })(\mathrm{scc} / \mathrm{min})}
\end{aligned}
$$

$=\frac{10 \mathrm{r}(200 \mathrm{kbits}) / \mathrm{monh}}{(30 \mathrm{davs} / \mathrm{month})(24 \mathrm{hrs} / \mathrm{dat})(60 \mathrm{Min} / \mathrm{hr})(60 \mathrm{sec} / \mathrm{min}}$$$
=0.771605 \text { bits } / \mathrm{Sec}
$$$$
\text { Rate }_{3}=\frac{\text { PQbits } / \text { week }}{(\text { days } / \text { week })(\text { hrs } / \text { day })(\mathrm{min} / \text { hrs })(\mathrm{sec} / \mathrm{min})}
$$$$
=\frac{10 r(200 \text { kbits }) / \text { week }}{(7 d a n s / \text { week })(24 h r s / d(c))(60 \mathrm{~min} / \mathrm{Hr})(60 \mathrm{Sec} / \mathrm{Min})}
$$$$
=3.3068783 \mathrm{bits} / \mathrm{SeC}
$$$$
D_{R T}=\text { Rate }_{1}+\text { Rate }_{2}+\text { Rate }_{3}
$$

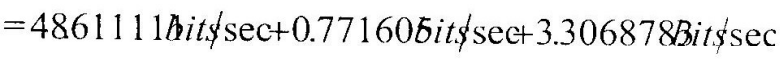

$$
=52.6895943 \text { bits } / \mathrm{sec}
$$

Considering High-Level Data-Link Control (HDLC) packet format with length $P_{L}$ bits per packet, the packets mean arrival rate $\lambda$ per meter is given by equation (5),

$$
\begin{aligned}
\lambda & =D_{T R} \cdot x \frac{1}{P_{L}} \\
& =52.6895943 \frac{\text { bits }}{\mathrm{sec}} \times \frac{1}{512} \frac{\text { packet }}{\text { bits }} \\
& =0.102909363 \frac{\text { puckets }}{\text { Sec }}
\end{aligned}
$$

Since powerline channel is the single channel (transmit one packet at a time) the transmission behaviour (service rate) is controlled by medium access control (MAC). The channel allows transmission of one packet at a time until all the packets from all meters have been served. The analysis of $\mathrm{MAC}$ is required to investigate if the MAC protocol is able to serve all packets generated by all customer meters within working hours (w.hours) period. The maximum number of customer meters per phase is 70. The investigation is based on traffic load generated by 70 - customer meters.

$$
\begin{aligned}
\mu=\frac{1}{P_{L}} & \\
& =\frac{1}{512} \text { Packet } / \mathrm{sec}
\end{aligned}
$$

The data traffic intensity per customer meter is given by equation (7).

$$
\begin{gathered}
\rho_{m}=\frac{\lambda}{\mu C}=\frac{52.6895943}{125 \times 10^{3}} \text { Erlang } \\
=0.000421516 \text { Erlang }
\end{gathered}
$$

\section{METERING TRAFFIC ANALYSIS}

The traffic Figures generated in each stage have been used to predict the network parameters. Due to the proposed network setup, two segments have been taken into consideration. These segments are from customer meters to bridging router and from bridging router to primary router.

\section{Customer Meters to Bridging Routers}

The traffic Figure generated by customer meters will be used to predict the MAC protocol required to serve the customer meters also the regenerative repeaters will be used in queuing delay analysis. 


\section{The Media Access Control Model}

The data traffic is modeled using queueing model of Figure 4, where both arrival process and service rate are modeled as Poisson and exponential processes respectively. $\lambda_{p \mid l}$ to $\lambda_{l n}$ are Poisson arrivals from different customer meters, $\mu$ is service rate and $C_{h}$ is the channel capacity. The arrival rate was increased while the service rate was controlled by either CSMA/CD, Pure ALOHA or slotted ALOHA

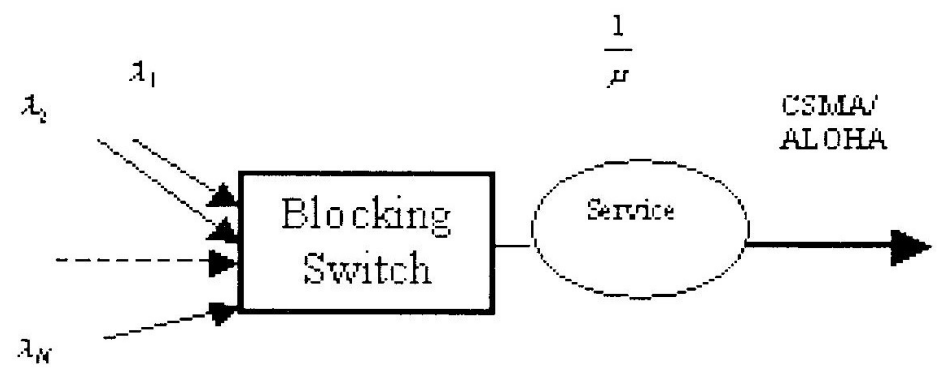

Figure 4: Metering Data packet queucing model

systems (Antonio, 2002) to observe which media access control (MAC) will be able to serve the traffic generated by maximum number of customers in each power line phase (70 Customer Meters is assumed).

Equation (8) gives the total traffic throughputs, where $\rho_{T}$ is the traffic intensity generated pes Customer meter and $N_{1}$ is tota number of customer meters pel phase. Equations (9), (10) and (11. present pure ALOHA $\left(S_{P, A}\right)$ slotted ALOHA $\left(S_{S A}\right)$ anc CSMA/CD $\quad\left(S_{C S M A / C D}\right)$ throughput respectively, whereby $\mathrm{G}$ is offered load and $a$ is the ratic between propagation time anc transmission time.

The packets received at the bus art processed immediately and if the bus is busy, the packets are blocked. The analysis of medium access control has been performed (MAC) at bridging router since the maximum number of traffic is flowing at this point.

$$
\begin{aligned}
& S=N_{t} \times \rho T \\
& S_{P A}=G \exp (-2 G) \\
& S_{S A}=G \exp (G) \\
& S_{(S W !(1)}=\frac{G e^{(-G i(1+2 a)}(1+G+a G(1+G+a G / 2)}{G(1+2 a)-\left(1-e^{-u(i)}\right)+\left(1+a(G) e^{-(i(1+a)}\right)}
\end{aligned}
$$

The required MAC will be used to perform the delay analysis for the packets and predict the traffic required to be handled by routers per phase for processing and setting the required token holding time for acceptable delay of packet data.

Figure 5 shows the responses of different communication protocols under different loads. It can be observed that at offered load of 0.0308 erlang the throughput of CSMA/CD can serve up to 70 customer meters, which is the maximum number of customer meters per phase, while Pure Aloha and slotted Aloha can serve up to 66 and 68 respectively. Hence, throughput of CSMA/CD is identified to be higher compared to other protocols and selected for use in this analysis.

\section{Modeling Regenerative Repeater Scenario in}

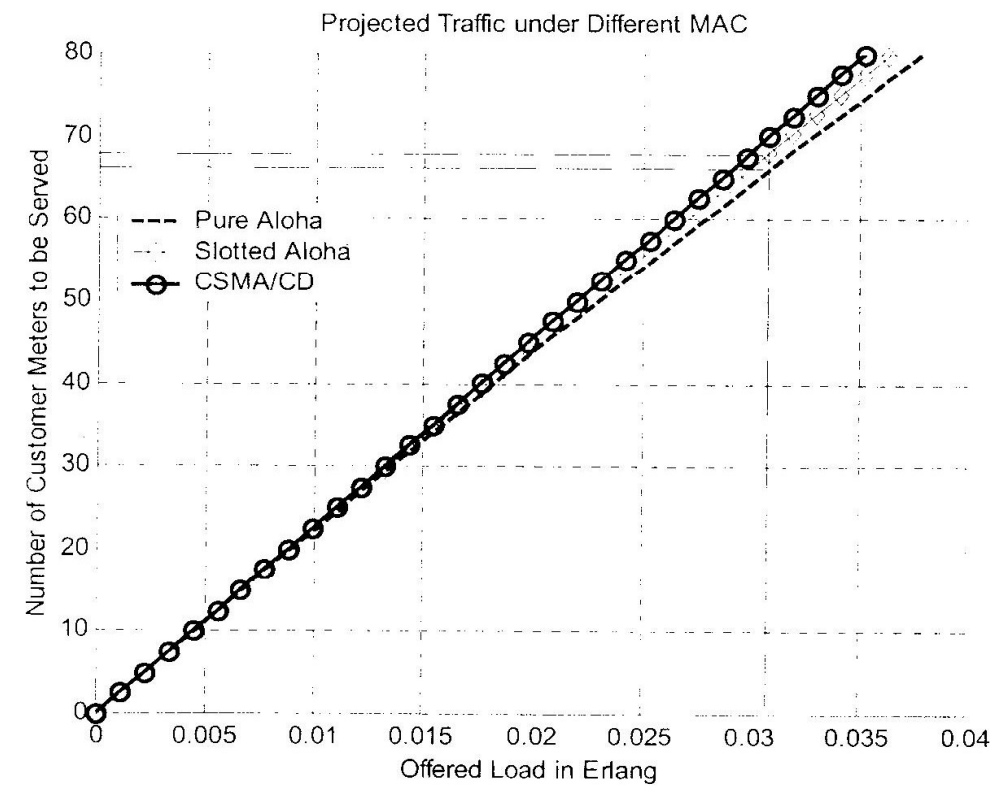

Figure 5: Projected traffic under different media access control 


\section{Powerline Network}

The signal attenuation in powerline network due to complex transfer characteristics and noise, it has have been established that two regenerative repeaters are necessary to enable transmission of data to bridging routers. The repeaters have been spaced by 400 meters in between due to the required $\mathrm{BER}$ performance and receiver sensitivity.

The diagrammatic model in Figure 6 is used in the delay analysis from customer meter to bridging Routers. The communication MAC-

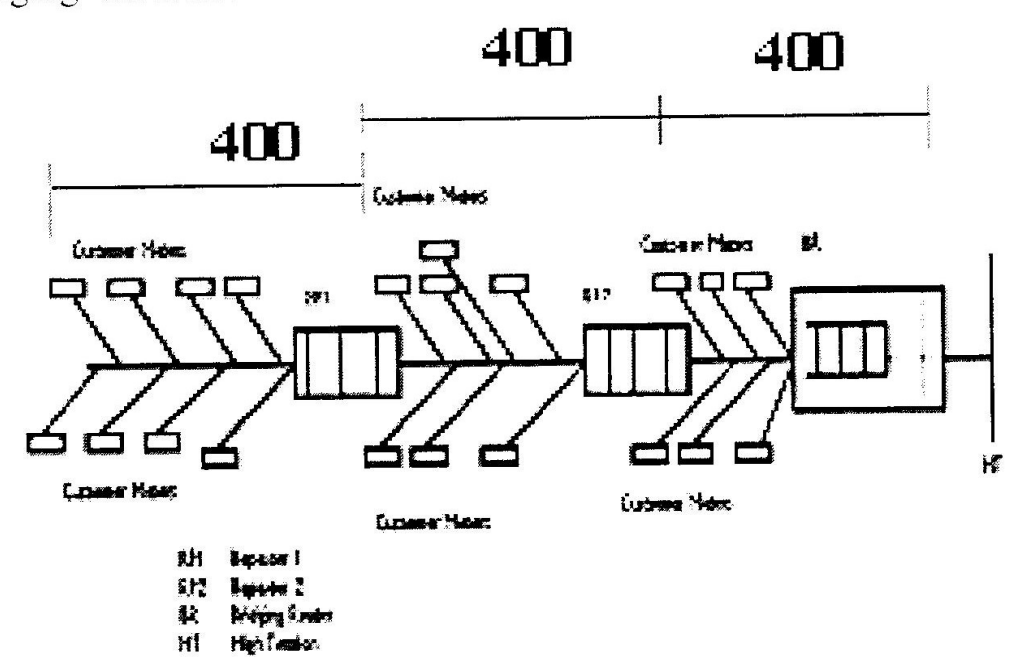

utilization) $\rho$ at each regenerative repeater has been calculated using equation (14).

$$
\begin{aligned}
& \mathrm{t}_{\mathrm{i}} / \mathrm{T}=\rho \frac{\left[B+(4 c+2) a+5 a^{2}+4 a\left(2 c^{2}-1\right) u^{2}\right]}{2 i 1-\rho(1+(2 c+1) a\}}+1+2 c^{\prime} a(12) \\
& \left(1-e^{-2 t, r}\right)\left(\frac{2}{2}+2 a e^{-1}-6 a\right) \\
& -\frac{\rho}{2\left[F_{p}(\lambda) e^{-\rho x-1}-1+e^{-2, x t}\right]}+\frac{a}{2} \\
& F_{p}(\lambda)=e^{-\rho^{P}} \\
& \rho=\frac{N_{\max } d_{r}}{1200} \rho_{m}
\end{aligned}
$$

where $N_{\max }$ is the maximum number of customer meters per phase, $d_{p}$ is the distance where the repeater is located and 1200 meters is the maximum distance from farthest customer meters to Bridging router. $\rho_{m}$ is the traffic intensity per customer meter. The accumulated delays to repeaterl ( $R P 1)$, repeater2 (RP2), and bridging router (BR) are as shown in equations (15), (16) and (17) respectively. The distances between different repeaters are $400 \mathrm{~m}, 800 \mathrm{~m}$ and $1200 \mathrm{~m}$ as shown in Figure 4. The parameters $t_{1}, t_{2}$ and $t_{3}$ are delays at repeater 1 , repeter2 and bridging router respectively which have been obtained using equations (12) and (13), substituting in

Figure 6: Modeling of two hops from customer meters to bridging Routers in low voltage line.

protocol obtained is applied. Based on CSMA/CD the delay equation used is as shown in equation (12), where $a$ is the ratio between propagation time and packet transmission time. $T$ is the packet transmission time. $B$ is the ratio between average second moment to second moment of data transmission rate. The assumption made is that the packet has constant length whereby this ratio is taken to be unity. Since the consideration is for constant length. $F_{\nu}(\lambda)$ in equation (12) is evaluated as in equation (13).

Basing on half duplex Time Division Duplex Mode (TDD) the traffic intensity in erlang (link equation (14), the respective traffic intensity at each station is given by (18).

$$
\begin{aligned}
& t_{R P 1}=t_{i} \\
& t_{R P 2}=t_{1}+t_{2} \\
& t_{B R}=t_{1}+t_{2}+t_{3} \\
& \rho=\frac{70\left(d_{r}\right)^{2}}{1200^{2}} \rho_{m}
\end{aligned}
$$

\section{Bridging Routers to Primary Router}

The traffic to the bridging routers is used to predict the delay required by packets to coup with other parameters such as data rate at high- 
tension grid, token rotation time etc. Figure 7 shows a cyclic queuing poling model (Higginbottom, 1980) (Cooper et al, 1969) adopted in this work applicable from bridging routers to primary router. This is the same as time division multiple access (TDMA). $\lambda_{R_{1}}$ to $\lambda_{R_{x}}$ are average packets arrival rates at bridging routers with buffers. In equation (18), $Q$ is the number of packets already queued at the routers, $t_{w}$ is the average waiting time at each station and $\lambda$ is equivalent to packet arrival rate. It is assumed that the packet arrival rate is the same

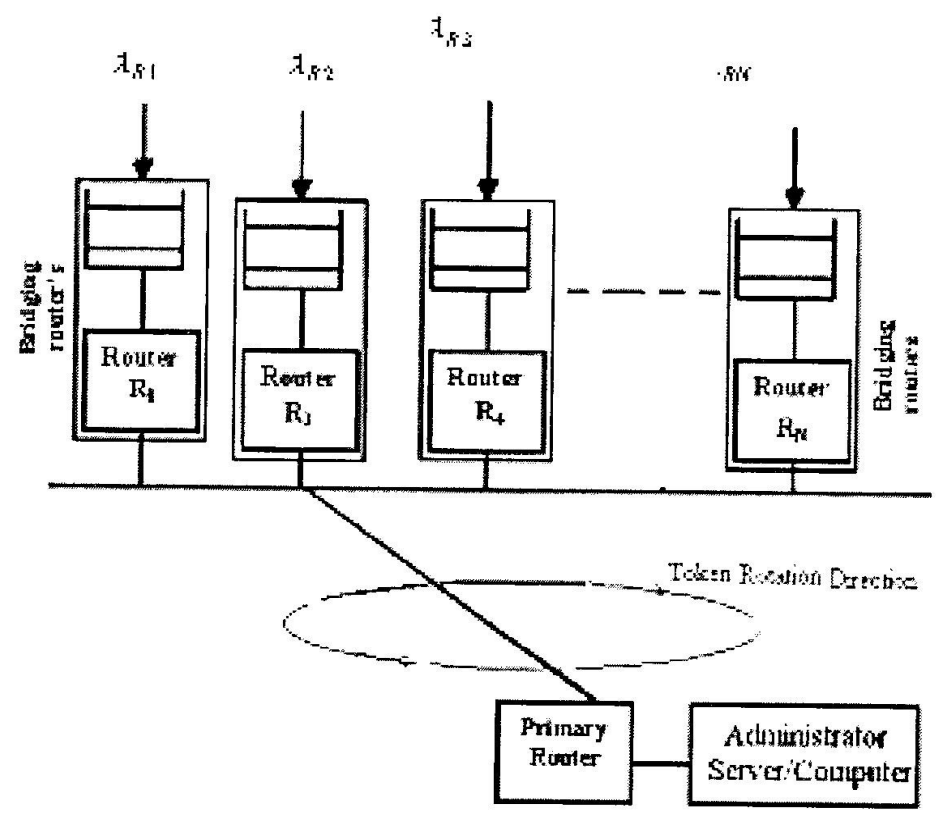

Figure 7: Cyclic model for collecting metering data from different bridging routers.

at each router in cyclic polling system.

$Q=\lambda t_{w}$

$t_{t r t}=\frac{N t_{\text {pass }}}{1-N \lambda t_{p}}$

$t_{r}=\frac{t_{t r t}}{2}$

In equation (20), N, $t_{p}$ and $t_{p a s s}$ are the number of routers in the entire network, propagation delay time and time constant that represents the time taken to pass the token respectively. In equation (21), $t_{r}$ and $t_{t r t}$ are the average residual token rotation time and token rotation time respectively. In equation (22), $d_{1}, P_{L}$ and $D_{R}$ are distance from data switch to bridging routers, distance from bridging router to primary router, packet length and data rate from bridging routers to primary routers respectively, $t_{d}$ represents queueing packet delay per routing station through powerline network and $v_{p}$ is a propagating velocity

$t_{d}=\frac{t_{r}}{1-\lambda t_{t r t}}+\frac{\rho_{L}}{D_{R}}+\frac{d_{1}}{v_{p}}$

Using Little's result (Kuo, 1981), (Harrison et $a l, 1993)$ the number of packet arrivals at bridging router, $Q$ is given by equation (19). The data packet received at all routers are queued and routed immediately upon receiving the token. The services are the same as exhaustive models (Cooper et al, 1969), (Cooper, 1970) but holding time dependant (HTD). On receiving token the routers processes all packets ready in the system and those arriving immediately during services and vacate the system when token holding time expires.

The relationship between the data rate at high-tension grid $D_{R H T}$ is obtained based on the number of stations serviced per line, and it is approximated by equation (23).

$$
D_{R H T}=N_{\max } D_{R}
$$

where $D_{R}$ is the data rate per customer meter. The variables we can investigate for a network parameters setup are:

Data rate $D_{R}$ : Data transmission rate of customer meter.

- Arrival process: $\lambda$

- Packet length: $\rho_{L}$

- Token rotation time: $t_{t r t}$ :

- Queuing packet delay $t_{p s}$ 


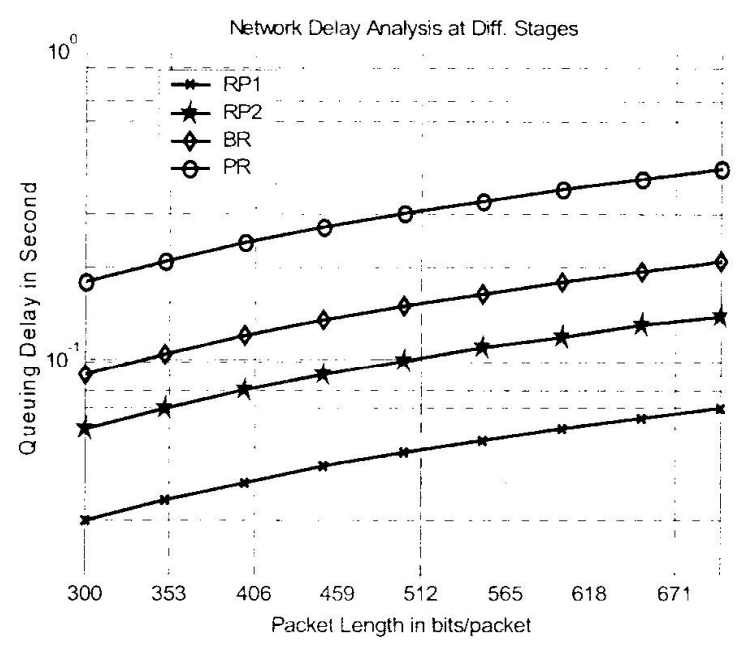

Figure 8: Packet queueing delay at different stages from customer meters to primary substation at $10 \mathrm{kbps}$.

The total packet queuing delay $t_{p s}$, from energy meter to a primary router is given in equation (24), where the parameters are obtained from equations (14) to (22).

$t_{p s}=t_{1}+t_{2}+t_{3}+t_{d}$

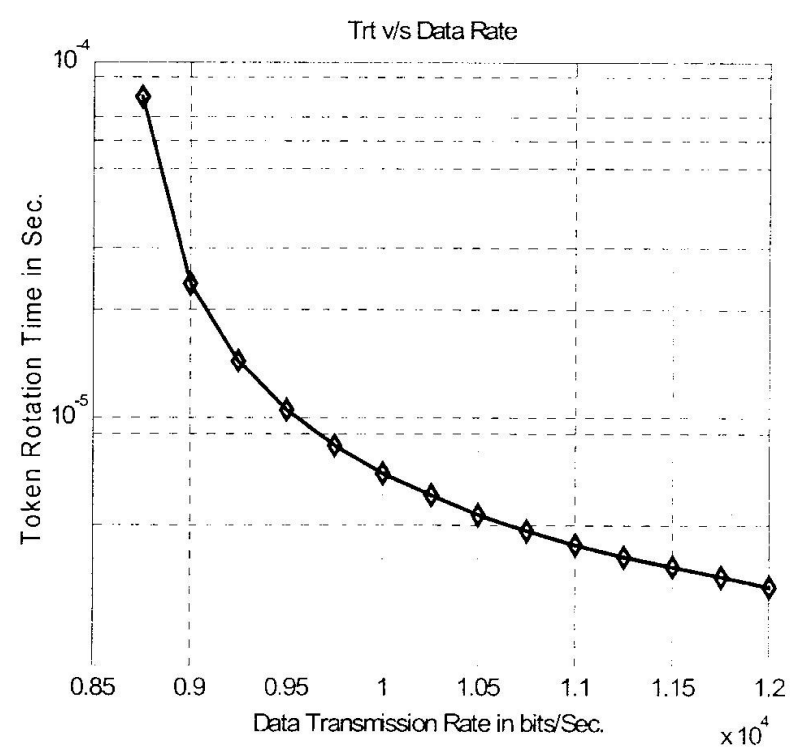

Figure 9: Dependence of packet queueing delay on Token rotation time at $512 \mathrm{bits} /$ packet

\section{SIMULATION RESULTS}

The interest is in two areas; the first is to set the required data transmission rate in the hightension side, the second is the impact of queuing delay elasticity in the packet. Figure 8 shows the dependence of queueing packet delay on packet

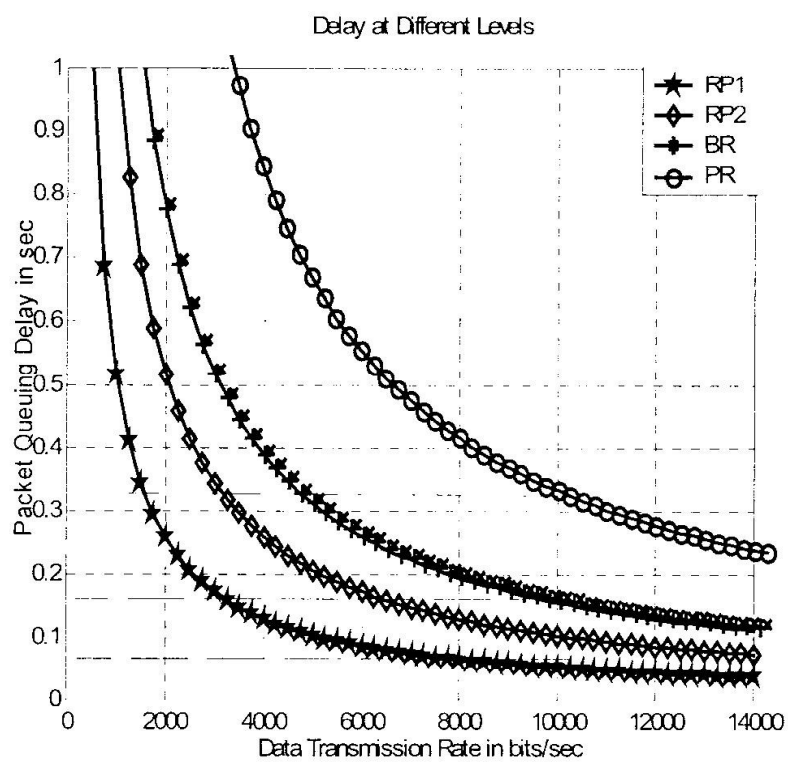

Figure 10: Dependence of packet queueing delay on data rate time at $512 \mathrm{bits} /$ packet 
length for different stages in powerline network. It can be observed that for packet length of $512 \mathrm{bits} /$ packet the queuing delays are $0.051 \mathrm{sec}$, $0.11 \mathrm{sec}, 0.16 \mathrm{sec}$ and $0.31 \mathrm{sec}$ from customer meter to first regenerative repeater, first regenerative repeater to second regenerative repeater, second regenerative repeater to bridging router and bridging router to primary router respectively. In addition, trends to first regenerative repeater and second regenerative repeater for $400 \mathrm{~m}$ the delays are constant, while from RP2 to BR is different due to the increase in traffic load

Figure 9 shows the dependence of data transmission rate on Token rotation time. It can be seen that for a packet with packet length of $512 \mathrm{bits}$ and data rate of $10 \mathrm{kbps}$ the token rotation time is $7 \times 10^{-6}$ seconds.

Figure 10 shows the dependence of queueing delay on data transmission rate for power grid network. It can be observed that for data transmission rate of $10 \mathrm{kbps}$ the queuing delays are $0.051 \mathrm{sec}, 0.11 \mathrm{sec}, 0.16 \mathrm{sec}$ and $0.31 \mathrm{sec}$ from customer meter to first regenerative repeater, first regenerative repeater to second regenerative repeater, second regenerative repeater to bridging router and bridging router to primary router respectively. The results obtained in Figure 10 are similar with the results obtained in Figure 8.

\section{CONCLUSION}

It has been realized that $\mathrm{CSMA} / \mathrm{CD}$ is a MAC protocol to be used from customer meter to bridging routers at distribution transformer. From bridging routers at distribution transformers to primary router at primary substations, token bus MAC protocols have to be used. From the data obtained, the selected parameters are data transmission rate of $10 \mathrm{kbps}$, token rotation time is 7 microseconds and Packet length is $512 \mathrm{bits} /$ packet. All these parameters will be used in network setup.

\section{ACKNOWLEDGEMENT}

The authors would like to acknowledge the sponsorship of Sida/SAREC through capacity building project at the prospective College of Engineering and Technology, University of Dar es Salaam.

\section{REFERENCES}

1. ANSI/IEEE Std, (1985), "Local Area Networks ANSI/IEEE standard Draft", International Standard Token Passing Bus access Method, 1985.

2. Antonio, L. and Antonella, M. (2002), "Designing the Internetworking of Terrestrial and Satellite IP-based Networks", IEEE Comms. Magazine, Feb. 2002, pp. 136-144.

3. Anatory J., Mvungi, N.H. and Kissaka, M.M. (2003), "Performance Evaluation of Different MAC Protocols for IP based Powerline Communication Networks in Developing Countries", Proceedings of $7^{\text {th }}$ International Symposium of Powerline Communication and Application, Kyoto, Japan, March $26^{\text {th }}$ to $28^{\text {th }} 2003$, pp. $160-166$.

4. Anatory J., Mvungi, N.H. and Kissaka, M.M. (2002), "Powerline Network: The Solution of Access to Non urban areas and links to Cellular, PSTN and PSDN', Conference Proceeding of the $4^{\text {th }}$ Annual conferences of Eastern African Power, Mining \& Telecoms Conference \& Exhibition $9^{\text {th }}-11^{\text {th }}$ September, Royal Palm Hotel, Dar es Salaam, Tanzania, 2002, pp.196-225.

5. Anatory J., Mvungi, N.H. and Kissaka, M.M. (2002), "Analysis of Powerline Channel Model for Communication from Primary Substation Node to End-Users", Accepted for Publication in Iranian Journal of Electrical and Computer Engineering, Tehran, Iran

6. Anatory, J., "Investigation of appropriate Technology for remote monitoring of Electrical Power Consumption in Tanzania", thesis submitted in fulfillment of Master of Science in Engineering (Electrical) of University of Dar Es Salaam, November, 2003. 
7. Cooper, R.B., "Queues Served in Cyclic Order Waiting Time", Bell System Technical Journal, Vol. 49, 1970, pp. 399-413.

8. Seger, A. K., "Energy Utilities around the World Address this Growing Problem", Metering International Magazine Archive, Issue 1, 2002, pp. 20.

9. Cooper R.B and Murray G, "Queues Served in Cyclic Order", Bell System Technical Journal. Vol. 48 pp 675-689, 1969

10. Harrison, P. G. and Patel, N. M. , "Performance Modeling of Communication Networks and Computer Architectures, International Computer Science, "AddisonWesley Publishing Company-1993.

11. Kuo F. F., "Protocols \& Techniques for Data Communication Networks", Prentice-Hall Series in Computer Applications in Electrical Engineering 1981.

12. Onunga, O. J. and Donaldson, W. R. (1989), "Personal Computer Communications on Intra Building Powerline LAN's Using CSMA with Priority Acknowledgements", IEEE Journal on Selected Area in Communications, Vol.7, No. 2, pp 180-191, Feb. 1989.

13. Grabowski, K. and Hagenhaus, L. (1989), "Traffic Models for ISDN with Integrated Packet switching, Tele-traffic Science for New Cost Effective Systems, Networks And Services", ITC-12, Elsevier Science Publisher B.V (North Holland) IAC, 1989 